\title{
Flow over Heated Terrain. Part I: Linear Theory and Idealized Numerical Simulations
}

\author{
N. ANDREW CROOK \\ National Center for Atmospheric Research, Boulder, Colorado \\ DONNA F. TUCKER \\ Department of Geography, University of Kansas, Lawrence, Kansas
}

(Manuscript received 30 March 2004, in final form 14 January 2005)

\begin{abstract}
The flow past heated topography is examined with both linear and nonlinear models. It is first shown that the forcing of an obstacle with horizontally homogenous surface heating can be approximated by the forcing of an obstacle with surface heating isolated over the obstacle. The small-amplitude flow past an obstacle with isolated heating is then examined with a linear model. Under the linear approximation, the flow response to heated topography is simply the addition of the separate responses to thermal and orographic forcing. These separate responses are first considered individually and then the combined response is examined. Nondimensional parameters are developed that measure the relative importance of thermal and orographic forcing. Nonaxisymmetric forcing is then considered by examining the flow along and across a heated elliptically shaped obstacle. It is shown that the low-level lifting is maximized when the flow is along the major axis of the obstacle.

The linear solutions are then tested in a nonlinear anelastic model. The response to a heat source and orography are first examined separately. Good agreement is found between nonlinear and linear models for the individual responses to thermal and orographic forcing. The case of uniformly heated flow past an obstacle is then examined. In these simulations, the thermal response is isolated by subtracting the orographic-only response from the full thermal-orographic response. The numerical simulations are able to capture the main features of the thermal response. Finally, numerical simulations of the flow along and across an elliptically shaped heated obstacle are examined, where it is verified that the lifting is maximized when the flow is along the major axis of the obstacle.

These results are extended in Part II of this study to examine the moist convective response to flow over both idealized terrain and the complex terrain of the Rocky Mountains of the United States.
\end{abstract}

\section{Introduction}

One of the most prevalent mechanisms for the generation of moist convection is the convergence of boundary layer air. Observational studies dating back to the Thunderstorm Project of the 1940s have indicated that storm development is often preceded by lowlevel convergence (see, e.g., Byers and Braham 1949). Convergence in the boundary layer can be produced by a number of mechanisms such as differential heating (e.g., at coastlines or land surface variations) or the baroclinicity associated with density currents and cold

Corresponding author address: N. Andrew Crook, National Center for Atmospheric Research, P.O. Box 3000, Boulder, CO 80307-3000.

E-mail: crook@ucar.edu fronts. Topography can also produce lifting at low levels, either by forced lifting as the flow impinges on the topography or baroclinically if the terrain is heated (Banta and Barker Schaaf 1987). These two mechanisms are the subject of the present study.

The adiabatic response to flow over topography has been studied extensively over the last $50 \mathrm{yr}$ or so. One of the reasons for this is that, unlike many flows in the atmosphere, the forcing is relatively straightforward to specify. Analytical solutions for flow over two-dimensional topography date back to the 1940s (Queney 1948). The flow past a three-dimensional obstacle was first examined by Wurtele (1957) and Crapper (1959).

The response to an isolated heat source has also been well studied, although not as extensively as the orographic problem. In general, heat sources in the atmo- 
sphere are difficult to specify, being both transient and dependent on the flow field. Nevertheless, the solutions to fixed heating have been studied in two dimensions by Smith and Lin (1982) and in three dimensions by Lin (1986).

The combined response to orography and heating has been examined theoretically in only a handful of studies (e.g., Raymond 1972; Smith and Lin 1982; Reisner and Smolarkiewicz 1994; Tian and Parker 2003). Smith and Lin (1982) examined the flow response to a two-dimensional heat source and then added the solutions of Queney (1948) for flow past a two-dimensional ridge. Reisner and Smolarkiewicz (1994) examined the three-dimensional flow past a heated obstacle with particular emphasis on the flow past the islands of Hawaii. The focus of their study was the effect that heating has on the upwind stagnation point that develops in low Froude number flow past an obstacle. In the present study, the emphasis is on the circulations that develop in the lee of a heated mountain.

The outline of this study is as follows. In section 2 , we develop a linear model of the effects of orographic and thermal forcing. In section 3 we examine solutions of the linear model. In section 4, we test the predictions of linear theory for dry adiabatic flow using a numerical model with idealized terrain and heating. Conclusions are given in section 5 . In the second part of this study (Tucker and Crook 2005, hereafter Part II) we will examine the moist convective response to heated flow over both idealized and complex topography.

\section{Linear theory}

We first examine the case of flow past an obstacle with a surface heating rate that is constant in the horizontal. A schematic of this flow is shown in Fig. 1a. The terrain height is given by $H_{\mathrm{xy}}(x, y)$, with a maximum height of $H$. We make the linear approximation that the mountain height, $H_{\mathrm{xy}}(x, y)$, is infinitesimally small and apply boundary conditions at $z=0$. To model the effects of surface heating, we assume a heating profile that is maximized at the surface (with value $Q_{o}$ ) and then decays exponentially with an $e$-folding depth of $D$ above the surface. The three-dimensional distribution of heating is then given by

$$
Q_{\mathrm{xyx}}=Q_{o} e^{-\left(z-H_{\mathrm{xy}}\right) / D} .
$$

We now make the assumption that the heating over the plains surrounding the obstacle is not dynamically important, since it does not generate any baroclinicity. We thus subtract this horizontally homogenous heating rate, $Q_{o} e^{-z / D}$, from the full heating function to obtain
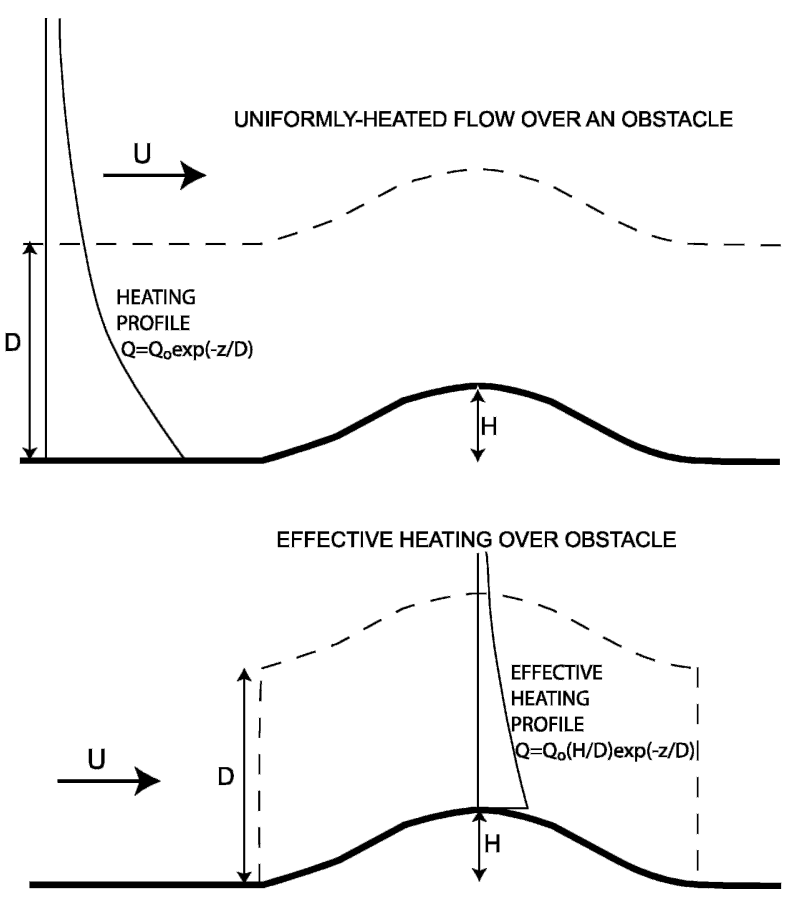

FIG. 1. (a) Schematic of flow with horizontal-uniform heating (heating rate $Q_{o}$, depth $D$ ) past an obstacle of height $H$. (b) Equivalent flow past an obstacle of height $H$ with heating of depth $D$ isolated over the obstacle. The effective heating rate is reduced by a factor $H / D$.

$$
Q_{\mathrm{xyx}}=Q_{o} e^{-z / D}\left(e^{H_{\mathrm{xy}} / D}-1\right) .
$$

We note that since we are making the linear approximation that $H_{\mathrm{xy}}(x, y)$ is infinitesimally small, there is no contribution from the region below $H_{\mathrm{xy}}(x, y)$. If the heating depth, $D$, is finite, $H_{\mathrm{xy}}(x, y) / D$ is infinitesimally small and (2) becomes

$$
Q_{\mathrm{xyx}}=Q_{o} \frac{H_{\mathrm{xy}}(x, y)}{D} e^{-z / D}
$$

Thus, the effect of constant surface heating over an obstacle can be approximated by surface heating, which is isolated over the obstacle and has the same functional form as the terrain height; see Fig. 1b. Note that the dynamically important, or effective, heating has a maximum value, $Q_{o}^{\text {effective }}$, that is reduced by a factor of $(H / D)$ compared to the constant heating rate, that is,

$$
Q_{o}^{\text {effective }}=Q_{o} \frac{H}{D}
$$

We now develop a linear model of the atmospheric response to combined orographic and isolated thermal forcing. The development here follows the work of Reisner and Smolarkiewicz (1994) who examined axi- 
symmetric orographic and thermal forcing. In the present study, we will relax the assumption of axisymmetry and examine forcing that is stretched either along or across the flow. Equations (5)-(15) follow that of Reisner and Smolarkiewicz (1994) and are presented here for the sake of completeness.

To simplify the analysis we assume that the flow is steady state, Boussinesq, hydrostatic, and incompressible. We further assume that the upstream velocity, $U$, and Brunt-Väisälä frequency, $N$, are constant in the vertical. The governing equations for small-amplitude flow $(u, v, w)$ forced by a heat source $Q$ are then

$$
\begin{aligned}
U u_{x} & =-\phi_{x}, \\
U v_{x} & =-\phi_{y}, \\
\phi_{z} & =b, \\
u_{x}+v_{y}+w_{z} & =0, \\
U b_{x}+w N^{2} & =\frac{Q g}{\theta_{o}},
\end{aligned}
$$

where $\phi$ is the perturbation pressure and $\theta_{o}$ is the mean-state potential temperature. We define the displacement, $\eta$, by

$$
w=U \eta_{x}
$$

and specify the heating function given in Eq. (3).

The functions $Q$ and $\eta$ are then represented by their Fourier integrals:

$$
\begin{gathered}
\eta(x, y, z)=\int_{-\infty}^{\infty} \int_{-\infty}^{\infty} \hat{\eta}(k, l, z) e^{i(k x+l y)} d k d l, \\
Q(x, y, z)=\int_{-\infty}^{\infty} \int_{-\infty}^{\infty} \hat{Q}(k, l, z) e^{i(k x+l y)} d k d l .
\end{gathered}
$$

Combining Eqs. (5)-(9) leads to the following wave equation for $\hat{\eta}$ :

$$
\hat{\eta}_{\mathrm{zz}}+m^{2} \hat{\eta}=-\frac{i \hat{Q} g\left(k^{2}+l^{2}\right)}{\theta_{o} U^{3} k^{3}} e^{-z / D},
$$

where

$$
m=\frac{N}{U} \frac{\left(k^{2}+l^{2}\right)^{1 / 2}}{k} .
$$

The general solution to (13) is

$$
\hat{\eta}(k, l, z)=A e^{i|m| z}+B e^{-i|m| z}-\frac{i \hat{Q} g\left(k^{2}+l^{2}\right) e^{-z / D}}{\theta_{o} U^{3} k^{3}\left[(1 / D)^{2}+m^{2}\right]} .
$$

The constants $A$ and $B$ are determined by specifying upper and lower boundary conditions on $\hat{\eta}$. At the lower boundary, the displacement must equal the height of the topography; that is, $\hat{\eta}(k, l, z=0)=$ $\hat{h}(k, l)$ where $\hat{h}$ is the inverse Fourier transform of the orography. The upper boundary condition is that energy propagates away from the region of forcing, which implies that $B=0$ for $k>0$. Applying these boundary conditions gives the following solution for the displacement, $\eta$ :

$$
\begin{aligned}
& \eta(x, y, z) \\
& =\int_{-\infty}^{\infty} \int_{-\infty}^{\infty} \hat{h} e^{i m z} e^{i(k x+l y)} d k d l \\
& \quad-i \frac{g}{\theta_{o} U} \int_{-\infty}^{\infty} \int_{-\infty}^{\infty} \hat{Q} \frac{e^{i(k x+l y)}\left(e^{-z / D}-e^{i|m| z}\right)}{k\left(\frac{U^{2}}{D^{2}} \frac{k^{2}}{k^{2}+l^{2}}+N^{2}\right)} d k d l .
\end{aligned}
$$

The first integral gives the terrain response (see Smith 1980) while the second integral gives the response to the heat source (see, e.g., Lin 1986). Note that because we have made the linear assumption, the total response to orographic and thermal forcing is just the sum of the individual responses. This in turn means that we can study the combined linear response to orographic and thermal forcing by examining separately the two responses. We should note that when nonlinear effects are taken into account it is not possible to make this separation. Also, as noted by Smith and Lin (1982), quadratic quantities such as momentum and energy fluxes cannot be separated into their individual components even under the linear assumption.

We assume the terrain has elliptically shaped contours of constant altitude and a bell-shaped variation as a function of distance from the center:

$$
h(x, y)=\frac{H}{\left[1+\left(r_{e} / a\right)^{2}\right]^{3 / 2}},
$$

where $r_{e}=\left[\left(x / e_{x}\right)^{2}+\left(y / e_{y}\right)^{2}\right]^{1 / 2}, a$ is the scale width of the obstacle/heating (width at which forcing is $1 / 2^{3 / 2}$ of the maximum value), and $e_{x} / e_{y}$ is the ellipticity.

A nondimensional version of (16) can be formed using the following variable change: 


$$
\begin{aligned}
& \tilde{x}=\frac{x}{a}, \quad \tilde{y}=\frac{y}{a}, \quad \tilde{z}=z \frac{N}{U}, \quad \tilde{k}=k a, \quad \tilde{l}=l a, \quad \tilde{\kappa}=\kappa a, \quad \text { where } \kappa=\left(k^{2}+l^{2}\right)^{1 / 2} \text { and } \tilde{\kappa}_{e}=\kappa_{e} a, \text { where } \kappa_{e}=\left(e_{x}^{2} k^{2}\right. \\
& \left.\tilde{x}=\frac{x}{a}, \quad \tilde{y}=\frac{y}{a}, \quad \tilde{z}=z \frac{N}{U}, \quad \tilde{k}=k a, \quad \tilde{l}=l a, \quad \tilde{\kappa}=\kappa a, \quad+e_{y}^{2} l^{2}\right)^{1 / 2}
\end{aligned}
$$

$$
\begin{aligned}
\frac{\eta(\tilde{x}, \tilde{y}, \tilde{z})}{H}= & \frac{1}{2 \pi} \int_{-\infty}^{\infty} \int_{-\infty}^{\infty} e^{-\tilde{\kappa}_{e}} e^{i(\tilde{k} \tilde{k}) \tilde{z}} e^{i(\tilde{k} \tilde{x}+\tilde{y})} d \tilde{k} d \tilde{l}-\frac{i \tilde{Q}_{o}}{2 \pi} \int_{-\infty}^{\infty} \int_{-\infty}^{\infty} \frac{e^{-\tilde{\kappa}_{e}}}{\tilde{k}\left(\frac{\tilde{k}^{2}}{\tilde{\kappa}^{2}}+\tilde{D}^{2}\right)} \times\left[e^{-\tilde{z} / \tilde{D}}-e^{i(\tilde{\kappa} / \tilde{k}) \tilde{z}}\right] e^{i(\tilde{k} \tilde{k}+\tilde{y})} d \tilde{k} d \tilde{l} \\
& \equiv I_{A}+I_{Q}
\end{aligned}
$$

where $\tilde{D}=(N D / U)$, which we will call a nondimensional heating depth, and $\tilde{Q}_{o}=\left(g Q_{o} a D / \theta_{o} U^{3}\right)$, which will be called the nondimensional heating rate. We note that $\tilde{Q}_{o}$ is the same as the nondimensional number developed by Smith and Lin (1982) to compare the effects of thermal and orographic forcing (taking into account the different definitions of $Q$ in the two studies).

The second integral in Eq. (18) indicates that the thermal response depends on the nondimensional heating depth, $\tilde{D}$. We now examine the response in two parameter regimes: small and large $\tilde{D}$ :

(a) Large heating depth, $\tilde{D}$ :

For large $\tilde{D},(18)$ reduces to

$$
\begin{aligned}
\frac{\eta(\tilde{x}, \tilde{y}, \tilde{z})}{H}= & I_{A}-\frac{i \tilde{Q}_{o}}{2 \pi \tilde{D}^{2}} \\
& \times \int_{-\infty}^{\infty} \int_{-\infty}^{\infty} \frac{e^{-\tilde{\kappa}_{e}}\left[e^{-\tilde{z} / \tilde{D}}-e^{i(\tilde{k} / \tilde{k}) \tilde{z}}\right]}{\tilde{k}} \\
& \times e^{i(\tilde{k} \tilde{k}+\tilde{y})} d \tilde{k} d \tilde{l} .
\end{aligned}
$$

Since the two integrals in (19) are of order one, the ratio of thermal to orographic response is proportional to $\tilde{Q}_{o} / \tilde{D}^{2}=\left(g Q_{o} a / \theta U D N^{2}\right)$.

(b) Small heating depth, $\tilde{D}$ :

For small $\tilde{D},(18)$ reduces to

$$
\begin{aligned}
\frac{\eta(\tilde{x}, \tilde{y}, \tilde{z})}{H}= & I_{A}-\frac{i \tilde{Q}_{o}}{2 \pi} \int_{-\infty}^{\infty} \int_{-\infty}^{\infty} \frac{e^{-\tilde{\kappa}_{e}}}{\tilde{k}}\left[e^{-\tilde{z} / \tilde{D}}-e^{i(\tilde{\kappa} \tilde{k}) / z}\right] \\
& \times\left[1+\frac{\tilde{l}^{2}}{\tilde{k}^{2}}\right] e^{i(\tilde{k} \tilde{x}+\tilde{y})} d \tilde{k} d \tilde{l} .
\end{aligned}
$$

Note that the second integral in (20) has a term that is proportional to $\left[1+\left(l^{2} / k^{2}\right)\right]=\left[1+\left(\lambda_{x}^{2} / \lambda_{y}^{2}\right)\right]$, where $\lambda_{x}$ $=2 \pi / k$ and $\lambda_{y}=2 \pi / l$. This term means that the response in terms of displacement will be greater if the thermal forcing has amplitude in modes that are stretched along the flow compared to across the flow (i.e., $\lambda_{x}>\lambda_{y}$ ). In other words, the response will be maximized when the flow is along the major axis of the heat source compared with across the source. In section $3 \mathrm{c}$, we will give a physical explanation for this dependence on forcing orientation.

We also note that the thermal forcing integral contains a term $\left[e^{-\tilde{z} / \tilde{D}}-e^{i(\tilde{\kappa} / \tilde{k} \mid)} \tilde{z}\right]$. For small $\tilde{z} / \tilde{D}$, this term is proportional to $\tilde{z} / \tilde{D}$. Hence the ratio of thermal to orographic response at small $\tilde{z} / \tilde{D}$ is proportional to $\tilde{Q}_{o} / \tilde{D}=\left(g Q_{o} a / \theta U^{2} N\right)$.

To summarize, for large heating depths the ratio of thermal to orographic response at low levels is proportional to $\tilde{Q}_{o} / \tilde{D}^{2}=\left(g Q_{o} a / \theta U D N^{2}\right)$; for small heating depths it is proportional to $\tilde{Q}_{o} / \tilde{D}=\left(g Q_{o} a / \theta U^{2} N\right)$. Hence, in both regimes, thermal forcing increases in importance as the heating rate, $Q_{o}$, and width, $a$, increase and as the flow, $U$, and stability, $N$, decrease. The reason for the dependence on $Q_{o}$ is fairly obvious. The dependence on the forcing width is due to the fact that the air spends a longer time in the heat source and can acquire a larger thermal perturbation. Similarly, as the flow, $U$, decreases, the air spends more time in the heat source. The dependence on stability arises because the heating has to be balanced by vertical advection of buoyancy. Hence, a given heating rate will produce more vertical displacement if the stability is reduced.

\section{Linear solutions}

\section{a. Axisymmetric orographic and thermal forcing}

In this section we examine solutions of the linear model developed in the previous section. We solve a discrete version of Eq. (18) by using 500 Fourier modes in both the $x$ and $y$ directions. We first examine the solutions for a circular mountain. Figure 2 shows the vertical velocity field for a flow with $\tilde{D}=1$ and $\tilde{Q}_{o}=1$. We normalize the vertical velocity by the velocity scale $w_{\text {scale }}=U(H / a)$ in order to keep the orographic response of order unity.

For orographic forcing, the vertical velocity has upward velocity on the upwind slope and downward velocity in the lee. Above the surface, gravity waves are produced with phase lines that tilt upstream with 

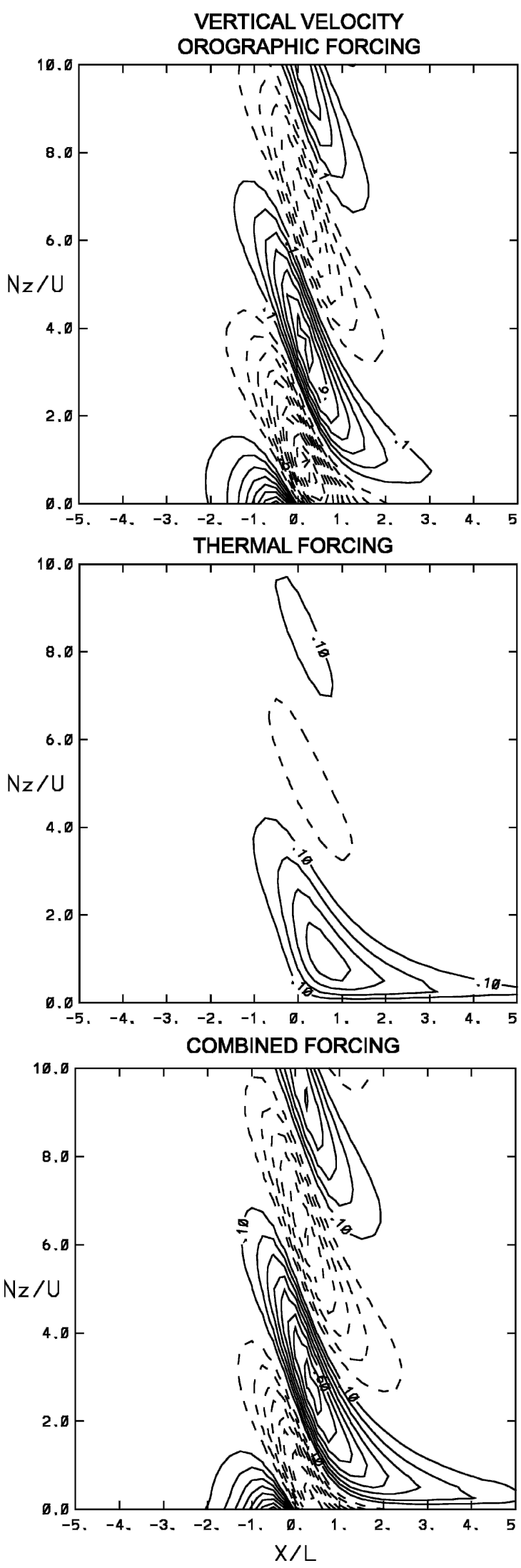

height, indicating upward energy transport. The vertical wavelength is $\tilde{z}=(N / U) z=2 \pi$. Note that the wave amplitude decreases with height, which is due to dispersion in the cross-stream $(y)$ direction.

For thermal forcing, the displacement and vertical velocity vanish at the surface. Above the surface, the heating causes weak downward velocity on the upwind side, with upward velocity only occurring in the lee. (This downward velocity is below the contour level in Fig. 2; however, it can be seen in Fig. 3 where we double $\tilde{Q}_{o}$.) This somewhat counterintuitive behavior was first explained by Smith and Lin (1982) who showed that it was a necessary condition for propagation of gravity wave energy in a stratified flow. In our example, since the heating occurs just above the surface, we can explain the initial downward velocity in the following manner. The heat source produces a region of low pressure at the surface and as the flow approaches this low pressure it accelerates, producing divergence. Since this divergence is occurring at the surface, it produces downward velocity above the surface. Upward velocity only occurs where the pressure gradient reverses and produces flow convergence. Note also that the upward velocity in the lee is significantly larger than the downward velocity ahead of the heat source. This is due to the fact that not only is there convergence in the $x$ direction, but also convergence in the cross-stream direction. The cross-stream convergence arises because the heating maximizes along the centerline of the heat source and decreases away from the centerline, which produces a cross-stream baroclinicity that in turn drives convergence in the cross-stream direction.

Thus, orography and heating produce opposite responses, with the former producing downward velocity in the lee of the orography, while heating produces upward velocity in the lee of the heat source. Whether upward or downward velocity occurs in the lee of heated terrain will thus depend on the relative importance of orographic to thermal forcing.

Figure $2 \mathrm{c}$ shows the combined response to orographic and thermal forcing, which is just the addition of the response shown in Figs. $2 \mathrm{a}$ and $2 \mathrm{~b}$. Since we are examining a case with $\tilde{Q}_{o}=1$ and $\tilde{D}=1$, the two responses should have approximately the same magnitude and thus should largely cancel out in the lee. How-

FIG. 2. Normalized vertical velocity for flow past heated terrain with $\tilde{D}=1, \tilde{Q}_{o}=1$. The first row shows the orographic response, the second row shows the thermal response, and the final row shows the combined response (sum of the first two rows). Contour interval equals 0.1 . 

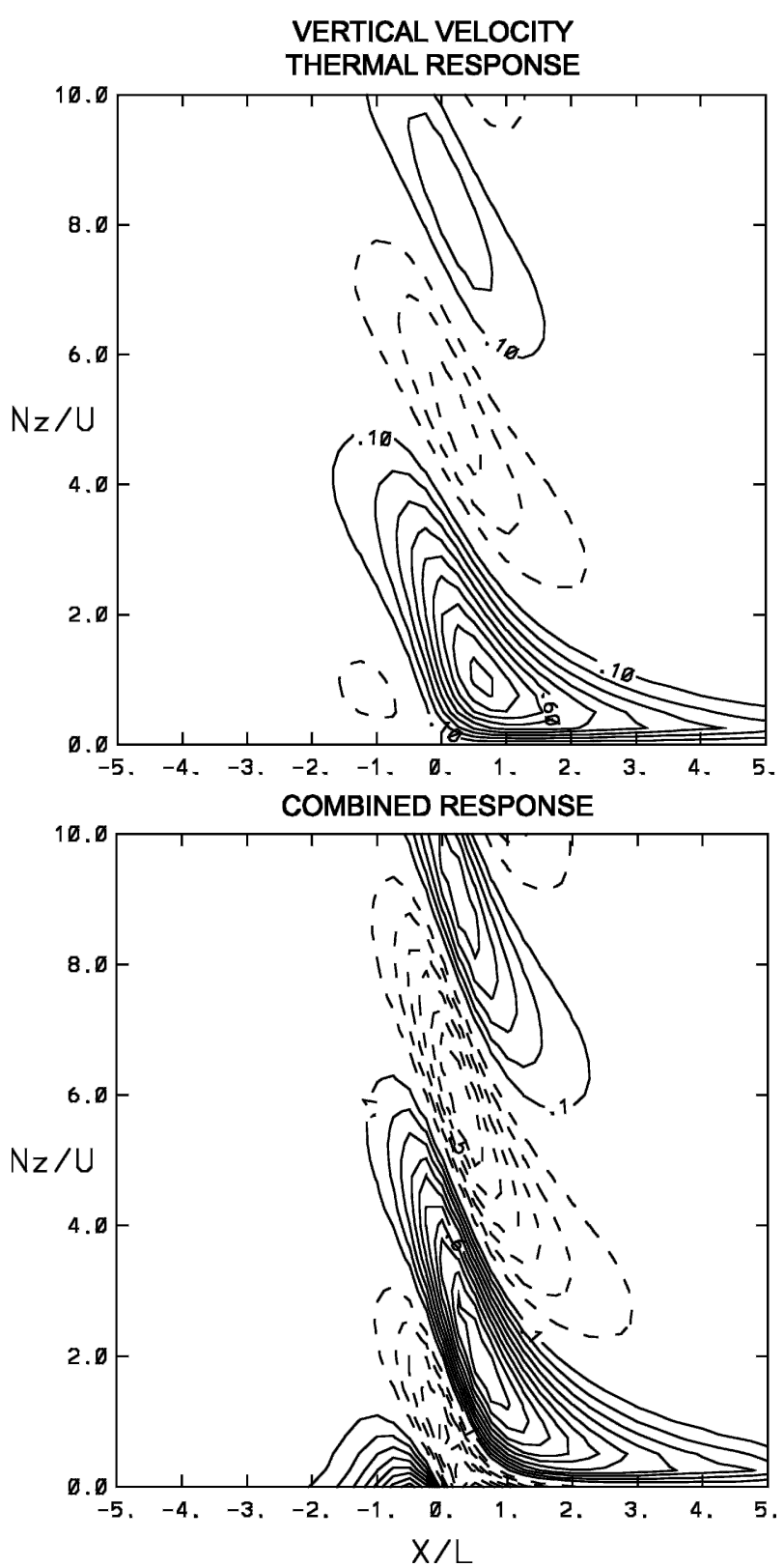

FIG. 3. Normalized vertical velocity in the (top) thermal response and (bottom) combined response for $\tilde{D}=1, \tilde{Q}_{o}=2$. Contour interval equals 0.1 .

ever, the thermal response has more amplitude at longer wavelengths compared to the orographic response. Mathematically, this is due to the wavenumber $k$ in the denominator of the second integral in (16). Physically, this can be explained by noting that orographic forcing only occurs in the vicinity of the orography, whereas heating can affect the flow some distance downstream through the advection of buoyancy perturbations away from the forcing. In turn this means that directly in the lee of the obstacle where short wave- lengths are important, the downward velocity produced by orography dominate. However, farther downstream of the heated obstacle where the longer wavelengths are important, the upward velocity produced by heating dominates.

We now examine ways to change the relative importance of thermal to orographic forcing. One way to increase the relative importance of the thermal forcing is to increase the nondimensional heating rate, $\tilde{Q}_{o}$. Figure 3 shows the thermal and combined response for $\tilde{Q}_{o}$ $=2, \tilde{D}=1$. (The orographic response is not shown since it is unchanged.) Far downstream of the heated obstacle, where the heating response dominates, the upward velocity has increased by a factor of 2 . However, close to the obstacle there is still orographically produced downward velocity in the lee.

Another way to increase the importance of thermal forcing is to decrease the depth of the forcing, $D$. To keep the same total heat input (which is proportional to $Q_{o} D$ ) we also need to increase $Q_{o}$, which in turn keeps $\tilde{Q}_{o} \equiv\left(g Q_{o} a D / \theta_{o} U^{3}\right)$ constant. In other words, the same amount of heat is put into the flow but over less depth. Figure 4 shows the thermal and combined response for $\tilde{D}=0.5, \tilde{Q}_{o}=1$. Again, the thermal response has increased by approximately a factor of 2 . In the previous section it was shown that for small $\tilde{D}$, the thermal forcing is proportional to $\tilde{Q}_{o} / \tilde{D} .^{1}$ Hence when $\tilde{D}$ is halved, at constant $\tilde{Q}_{o}$, the thermal forcing increases by a factor of 2. To explain physically the dependence on heating depth, we note that in section 2 we showed that horizontally uniform heating of depth $D$ over an obstacle of height $H$ has the same effect as isolated heating except with the heating magnitude reduced by a factor $H / D$. Hence, when the depth $D$ of the uniform heating is halved, at constant $Q_{o} D$, the total effective heat input, $Q_{o}^{\text {effective }} D$, increases by a factor of 2 .

\section{b. Nonaxisymmetric orographic and thermal forcing}

We now relax the assumption of axisymmetry by examining an elliptically shaped forcing feature. We first examine forcing that is stretched along the flow by setting $e_{x}=2$ and $e_{y}=0.5$ to give an ellipticity $\left(e_{x} / e_{y}\right)$ equal to four. The normalized displacement and vertical velocity is shown in Fig. 5 for a case with $\tilde{Q}_{o}=1.0$, $\tilde{D}=0.5$. As in Fig. 2, the top panel shows the orographic response, the middle panel shows the thermal response, and the bottom panel shows the combined

\footnotetext{
${ }^{1}$ We have verified from the full linear solutions that in the range $\tilde{D}=0.5 \rightarrow 1.0$ the thermal response is proportional to $\tilde{Q}_{o} / \tilde{D}$.
} 

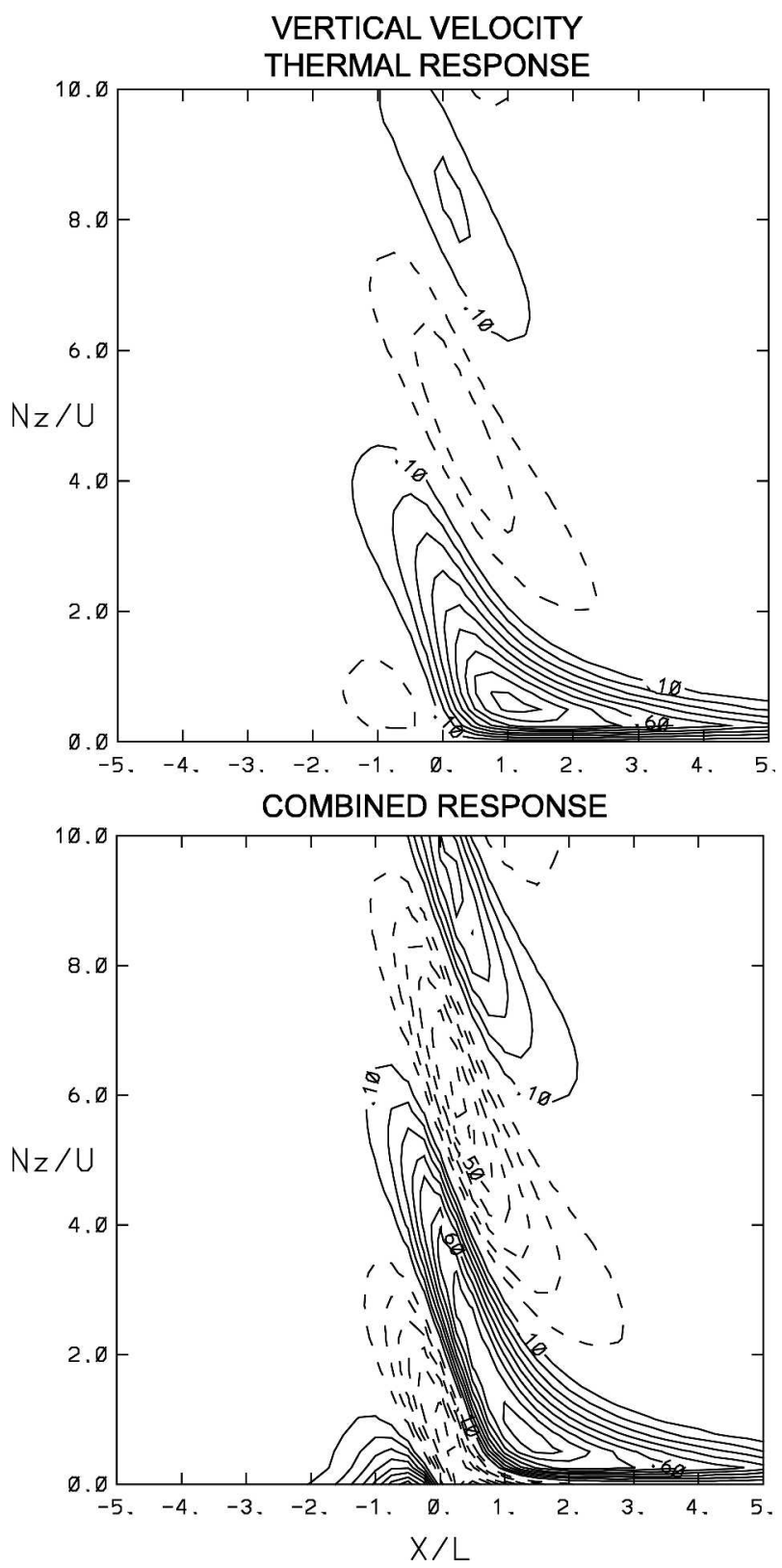

FIG. 4. Normalized vertical velocity in the (top) thermal response and (bottom) combined response for $\tilde{D}=0.5, \tilde{Q}_{o}=1$. Contour interval equals 0.1 .

response. The contour interval for both displacement and vertical velocity is 0.2 (compared to 0.1 in Figs. $2-4)$. As can be seen, the thermal response is significantly larger than the orographic response in this case. The upward velocity in the lee due to thermal forcing is significantly larger than the downward velocity caused by orography and consequently the combined response is upward everywhere in the lee, except for very close to the surface. Note also that there is very little gravity wave activity above $\tilde{z}=2$.
Figure 6 shows the response to forcing that is stretched across the flow (in the same format as before, contour interval $=0.2$ ). Now the orographic response is much larger than the thermal response. Consequently, in the combined response, downward velocity and displacement occurs in the lee (although farther downstream lifting returns). Note also that the gravity wave activity aloft is much stronger in this case.

\section{c. Physical explanation for response to nonaxisymmetric forcing}

Figure 7 is a schematic of the different responses to flow along or across a heated obstacle. The first/second row of Fig. 7 shows the response when the flow is along/ across the heated obstacle. When the flow is along the major axis of the heat source, a large cross-stream temperature gradient develops. This drives a cross-stream circulation with convergence and lifting along the centerline of the heat source. This effect is maximized when the flow is along the heat source and minimized when the flow is across the heat source.

The orographic response to flow along or across an obstacle is shown in the second column of Fig. 7. When the flow is along the major axis of the obstacle, the downward velocity forced by the lee slope is minimized, whereas it is maximized when the flow is across the obstacle.

To summarize, when the flow is along the major axis of a heated obstacle, the upward velocity forced by heating is maximized and the downward velocity in the lee forced by orography is minimized. Consequently, the maximum leeside lifting occurs when the flow is along the major axis of a heated obstacle.

\section{Numerical simulations}

We now examine numerical simulations of flow over heated terrain. We first examine the response to a heat source and orography separately and then examine the combined response. The simulations use the ClarkHall nonhydrostatic, anelastic, numerical model, Clark (1977). The model uses a terrain-following coordinate system, with free-slip boundary conditions used at the lower surface and a Rayleigh damping/Newtonian cooling layer specified in the upper third of the domain. The grid spacing is $0.1 a$ in the horizontal and $0.1(U / N)$ in the vertical. The domain size is $240 a$ in the $x$ (along flow) direction, $150 a$ in the $y$ (across flow) direction, and 6(U/ $N$ ) in the vertical. The low-level heating is applied with the same function used in the linear model, that is, $Q_{\mathrm{xyx}}$ $=Q_{o} e^{-\left(z-H_{\mathrm{xy}}\right) / D}$, where $H_{\mathrm{xy}}$ is the terrain height.

Before examining the numerical simulations, it is im- 

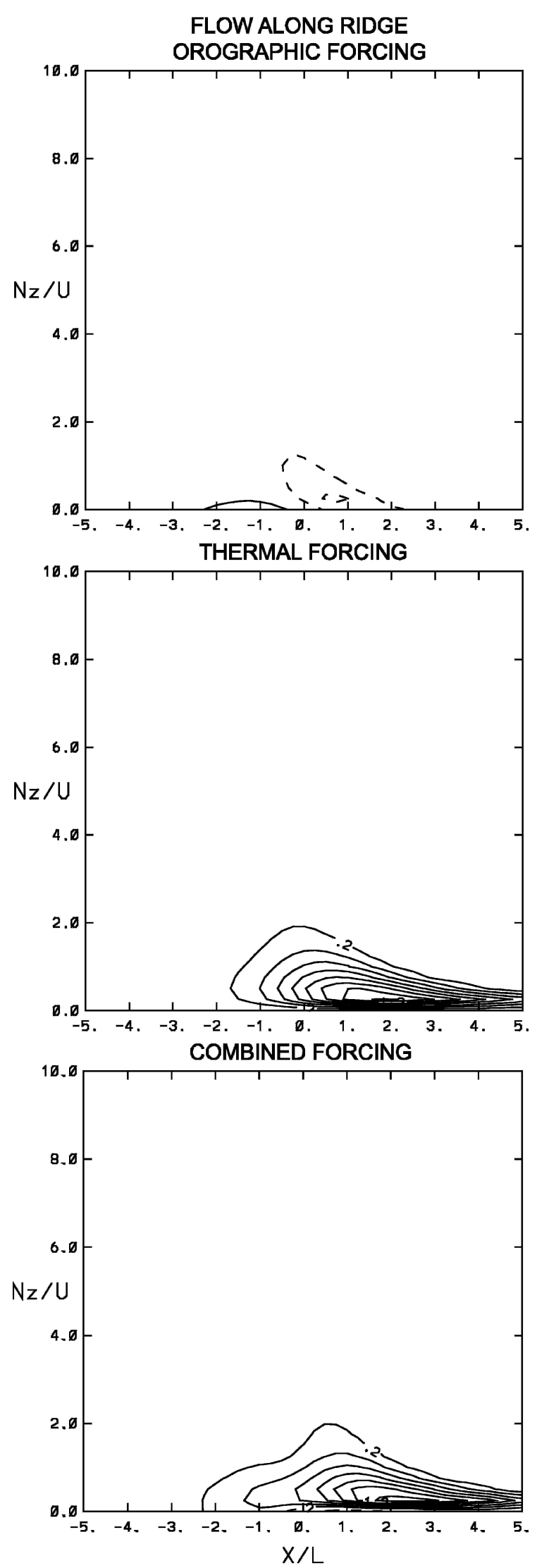

portant to determine the parameter regimes where nonlinear effects become important. For orographic forcing, the parameter that determines the degree of nonlinearity is the Froude number, $\mathrm{Fr}=U /(\mathrm{NH})$. Nonlinear effects become important for $\mathrm{Fr} \leq \mathrm{O}(1)$. For flow past an axisymmetric, bell-shaped obstacle, the flow reverses in the lee for $\mathrm{Fr} \leq 0.5$ (Smolarkiewicz and Rotunno 1989).

For flow past a heat source, there are two sources of nonlinearity. The first is when the magnitude of the perturbed flow approaches that of the mean-state flow. That nonlinearity is determined by the parameter $\tilde{Q}_{o}$. The other source of nonlinearity is when the perturbation temperature stratification produced by the heating approaches that in the mean-state stratification. If the heating produces a perturbation temperature stratification that exceeds the base-state stratification and of the opposite sign, then the flow will become unconditionally unstable and overturning can occur. Comparing the base-state stratification, $(\partial \theta / \partial z)=\left(\theta_{o} / g\right) N^{2}$, with the rate at which the stratification is reduced, $Q_{o} / D$, gives a time scale for this destabilization of $\left(\theta_{o} N^{2} D / g Q_{o}\right)$. Comparing this time scale with the advection time scale, $a / U$, indicates that the amount of destabilization that occurs in one advection time scale is equal to $(a Q g /$ $\left.N^{2} U D \theta_{o}\right)=\tilde{Q}_{o} / \tilde{D}^{2}$.

\section{a. Thermal and orographic effects separately}

We first examine the effects of orographic and thermal forcing separately. Figure 8 shows the flow over an axisymmetric obstacle with no heating. For this flow, Fr $=U /(N H)=10$ so nonlinear effects should be quite small. Furthermore, the nonhydrostatic parameter $U /(a N)=1 / 40$, so nonhydrostatic effects should also be negligible. The numerical results are shown in the top panel, and linear results are shown on the bottom panel. As can be seen, in general the numerical results match the linear solutions very well.

We now examine the flow past an isolated heat source, with no topography. To compare with the solutions for uniformly heated flow past topography, we define the isolated heat source using Eq. (3) and set $\tilde{Q}_{o}$ $=1, \tilde{D}=1$, and $H / D=0.2$. Figure 9 shows the (a) numerical solution and (b) linear solution for flow past an isolated heat source (no topography) at these parameter settings. [Note that Fig. $9 \mathrm{~b}$ is the same as Fig. $2 b$ except with a contour interval of 0.05.] As can be

$\leftarrow$

FIG. 5. Same as Fig. 2 except for flow along the major axis of an elliptically shaped forcing feature (ellipticity equal to four). The contour interval equals 0.2 . 

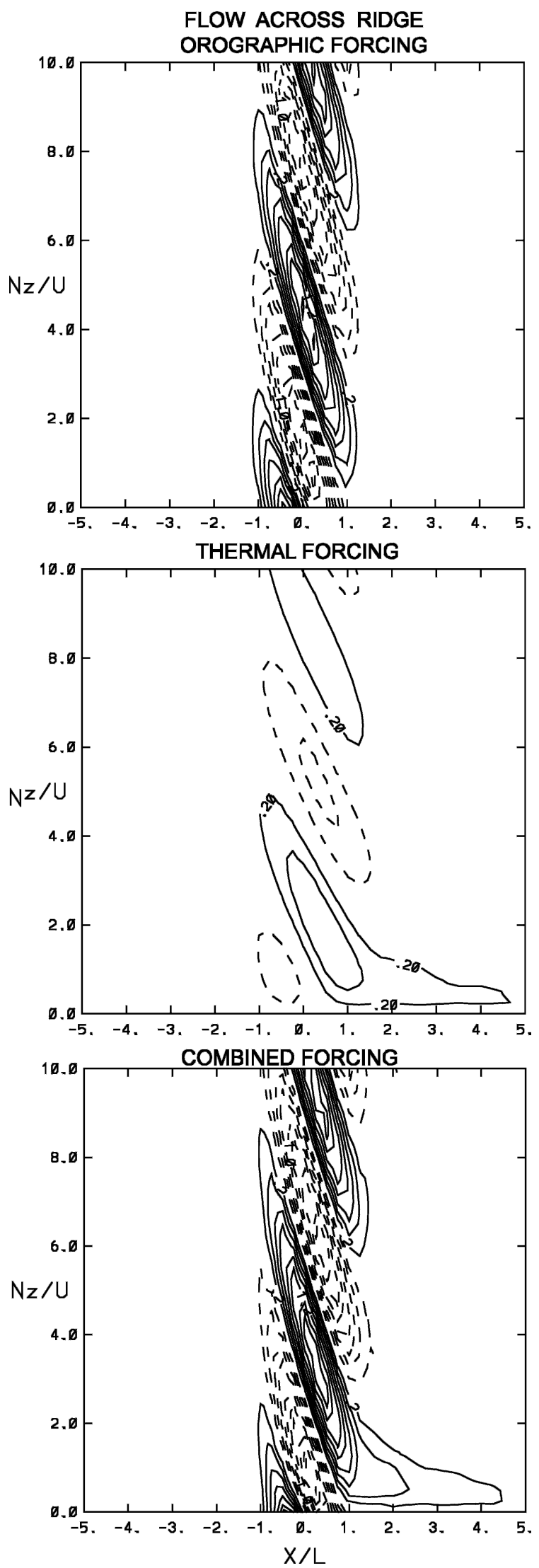

seen, the numerical results compare quite well with the linear solution. The numerical simulation captures the main updraft in the downstream half of the heat source as well as the downward motion upstream of the heat source. The wave amplitude does decay upward faster in the numerical simulations than in the linear solutions, which is most likely due to diffusion (both explicit and implicit) in the numerical model.

\section{b. Thermal and orographic effects combined: Flow past an obstacle with constant surface heating}

We now examine simulations of flow past an obstacle with a surface heating rate that is constant in the horizontal. In section 2, we argued that the constant heating over the plains surrounding the obstacle was not dynamically important since it does not produce any horizontal temperature gradients. However, the heating over the plains is thermodynamically important since it reduces the low-level temperature stratification with time. In other words, there is no steady-state solution for uniformly heated flow past an obstacle. However, a steady-state solution can be approached by setting the heating rate to be small. In the previous section, it was shown that the amount of destabilization that occurs in one advection time scale is equal to $\tilde{Q}_{o} / \tilde{D}^{2}$. By setting this value to be small, a steady-state solution can be approached before significant destabilization has occurred.

To isolate the thermal response in these simulations, a second experiment was performed of flow past the same obstacle but with no surface heating. To obtain the thermal response, the orographic-alone flow was then subtracted from the thermal-orographic flow, recalling that the two responses are additive in the linear limit.

In performing these simulations it was found that the heating rate could not be set too small or else it was difficult to isolate the thermal response. When subtracting the orographic-alone response from the thermalorographic response, any small noise in either simulation (e.g., from reflections off the upper boundary) would swamp the thermal response. We thus set $\tilde{Q}_{o} / \tilde{D}^{2}$ to be moderately small and ran the simulations for 10 time scales, $10 a / U$, to approach a steady state. To ensure that the heating did not reduce the low-level stratification to zero, extra stability was added to the initial temperature profile at low levels; the magnitude of this

$\leftarrow$

FIG. 6. Same as Fig. 5 except for flow across the major axis of an elliptically shaped forcing feature. The contour interval equals 0.2 . 
LOOKING VERTICALLY
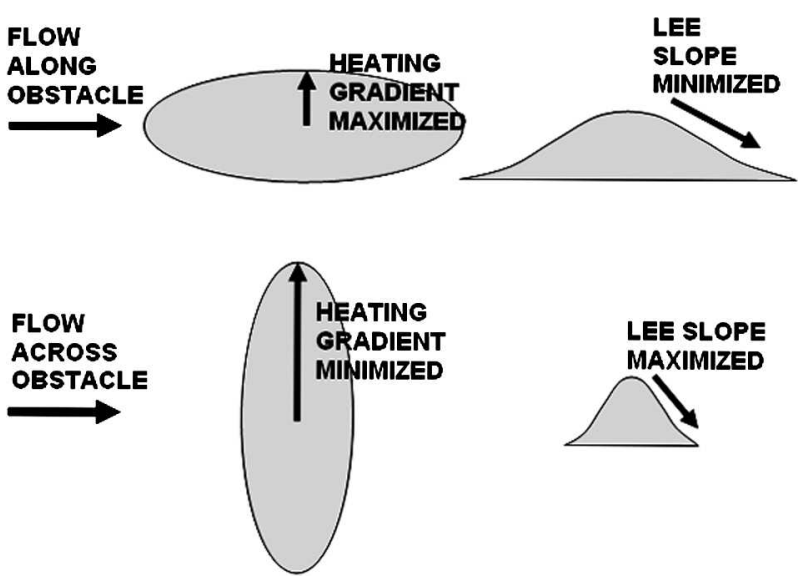

FIG. 7. Schematic showing the different forcing that occurs when the flow is along or across a heated obstacle.

extra stability was chosen so that the heating would remove it by the end of the simulation.

The result of this exercise is shown in Fig. 10 for the case of $\tilde{Q}_{o}=0.1, \tilde{D}=1$, and $H / D=1 / 5$. Figure $10 \mathrm{a}$ shows the vertical velocity from the numerical experiment (thermal-orographic response minus the orographic response) while Fig. 10b shows the thermal response in the linear model. As can be seen, the agreement is not as close as in the previous example with isolated heating. As mentioned above, since we are showing the difference between two nonlinear simulations, any small errors in either simulation can lead to large differences. Nevertheless, the numerical results capture the main features of the linear solution including the strength of the main updraft, descent ahead of the heat source (somewhat stronger in the numerical simulation) and the train of gravity waves above the heat source.

As discussed in section 3, one way to increase the relative importance of thermal forcing is to decrease the heating depth, $\tilde{D}$. Figure 11 shows the thermal response for a flow with the same parameters as in Fig. 10 , except with the heating depth decreased to $\tilde{D}=0.5$. As can be seen, the thermal response in both nonlinear and linear models increases by approximately a factor of 2. The agreement between nonlinear and linear solutions is similar to that in Fig. 10. Although there are clearly some differences, the numerical model captures the main features of the linear solution including the main updraft, descent ahead of the heat source, and gravity waves propagating aloft.

\section{c. Nonaxisymmetric orographic and thermal forcing}

We now examine simulations of flow past nonaxisymmetric orography. For these simulations, we have
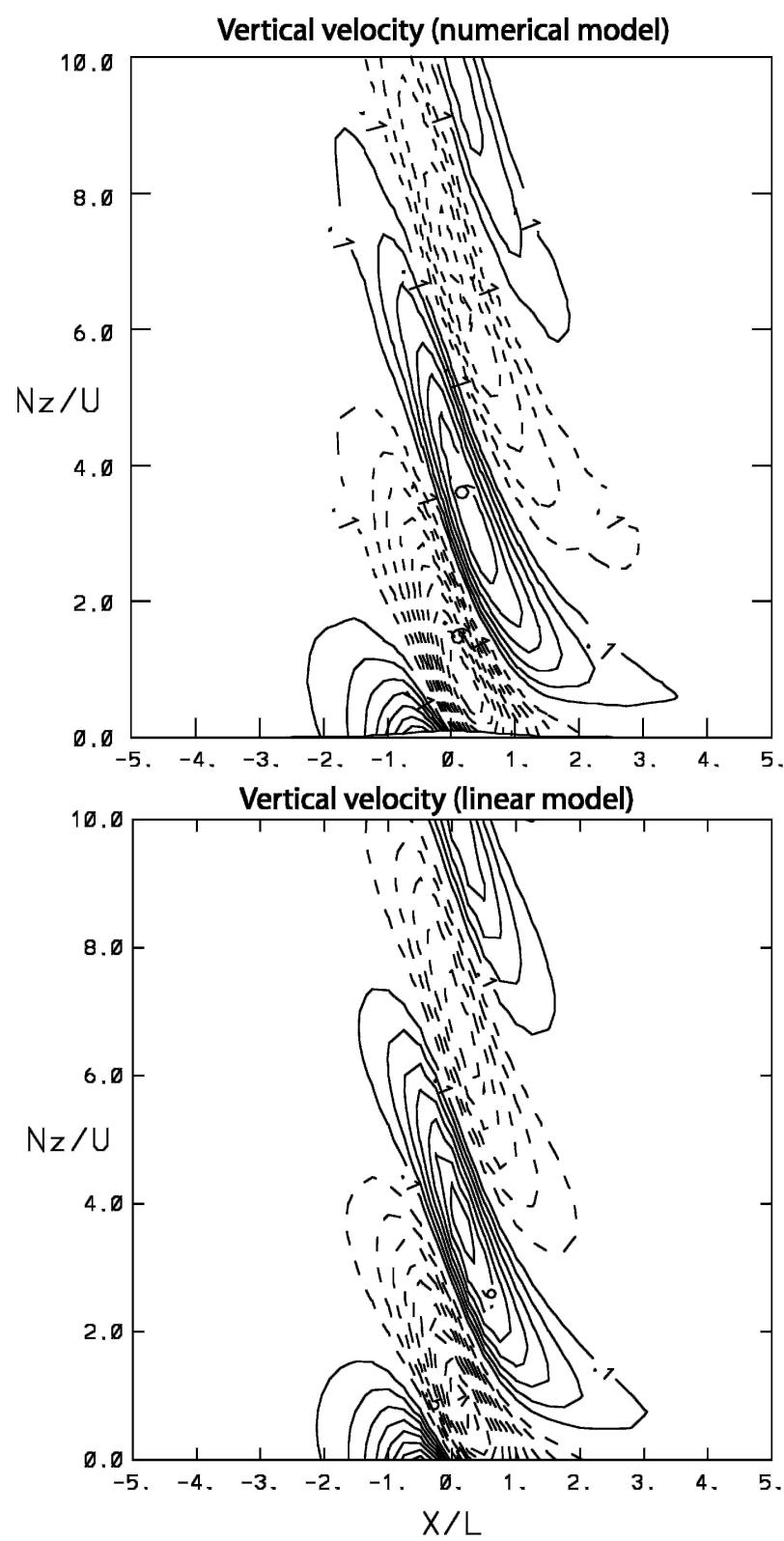

FIG. 8. Vertical velocity for flow over an axisymmetric obstacle at Froude number $F r=10$ from (a) the nonlinear model and (b) the linear model. Contour interval equals 0.1.

chosen an elliptically shaped obstacle with an ellipticity equal to four. For these experiments we plot the full thermal-orographic response at a moderate value of $\tilde{Q}_{o}$, since our main goal is to show the difference between flow along and across a heated ridge. Figure 12 shows the full response when the flow is (a) along and (b) across the major axis of the ellipse with $\tilde{Q}_{o}=$ 1.0 and $\tilde{D}=0.5$. The equivalent linear solution for the along-ridge flow is shown in Fig. 5c and in Fig. 6c for the across-ridge case. Despite the fact that $\tilde{Q}_{o}$ is 
VERTICAL VELOCITY (NUMERICAL MODEL)
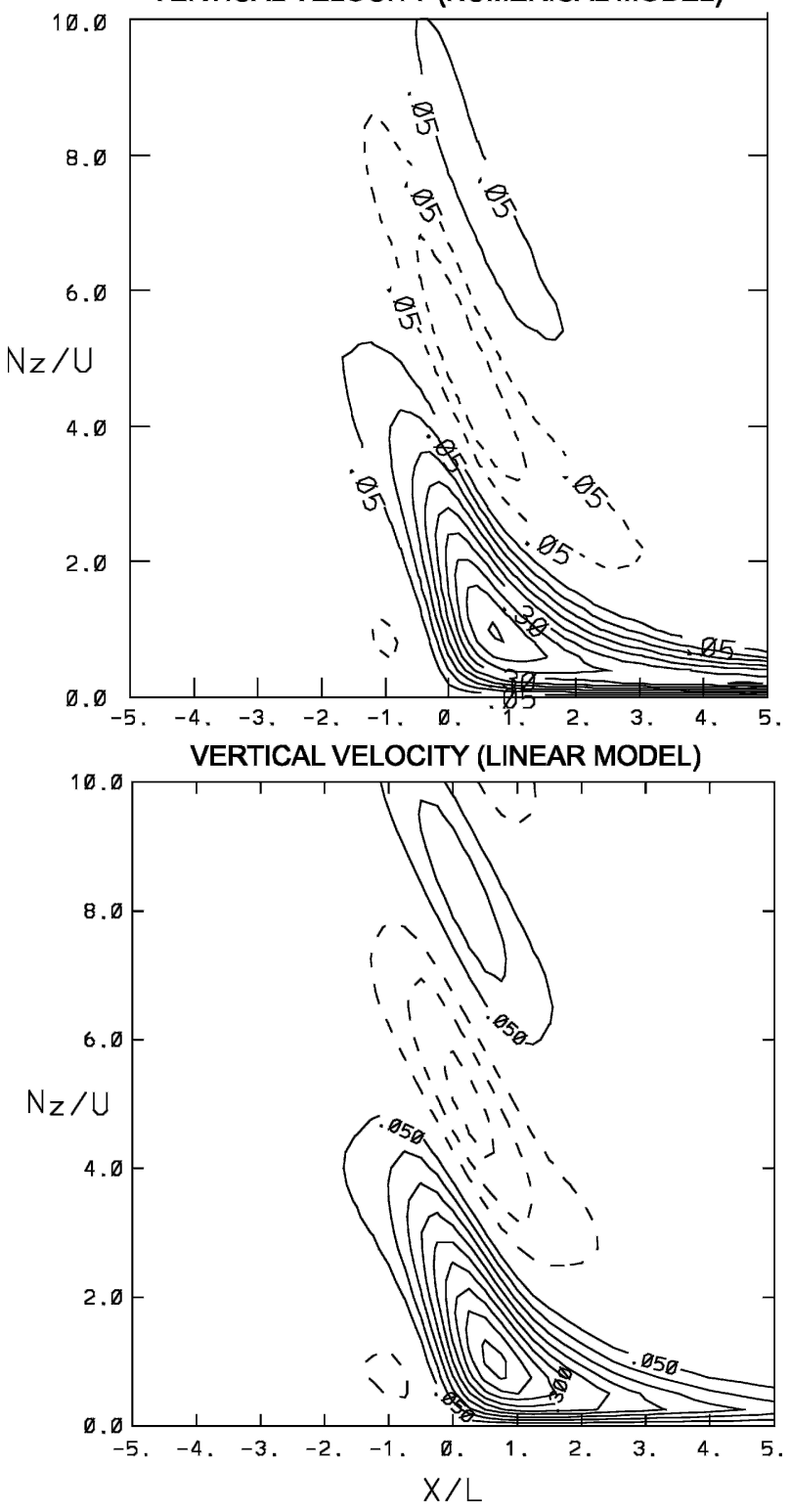

FIG. 9. Vertical velocity for flow over an isolated heat source with $\tilde{Q}_{o}=1, \tilde{D}=1, H / D=0.2$ from (a) the numerical model and (b) the linear model. Contour interval equals 0.05 .

not small, the level of agreement with the linear solutions is quite good. The most important point though is to compare the responses when the flow is along or across the major axis of a heated obstacle. When the flow is along the major axis, a single band of upward velocity develops on the lee slope of the ridge and there is very little gravity wave activity aloft. In contrast, when the flow is across the ridge, there is primarily downward motion on the lee slope with strong gravity wave activity above. In Part II we will show how these different responses have a strong effect
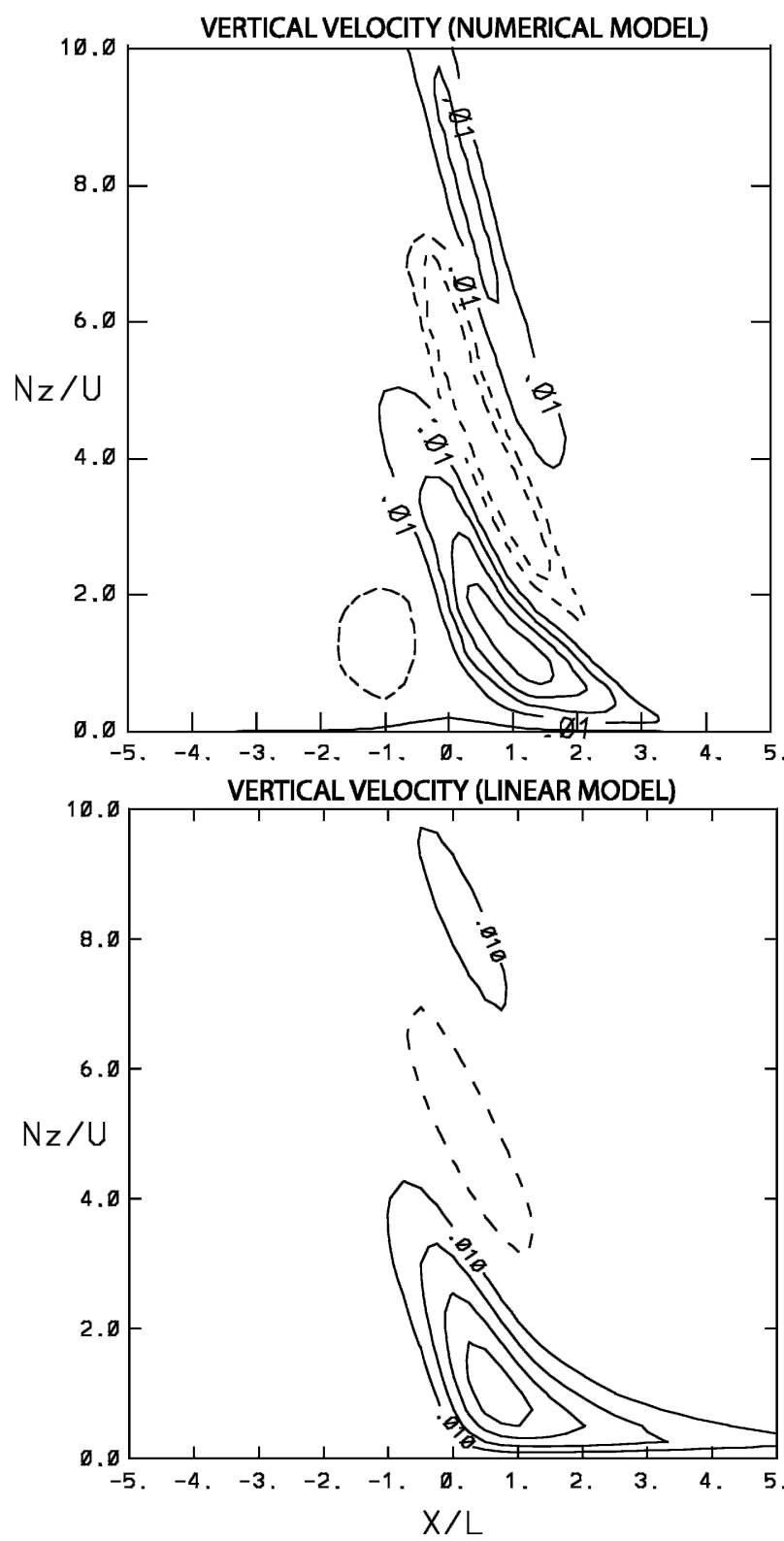

FIG. 10. Thermal response for flow over heated terrain with constant surface heating, $\tilde{Q}_{o}=0.1, \tilde{D}=1$. Thermal-orographic response minus orographic response from (a) numerical simulation and (b) linear solution.

on the development of moist convection over heated terrain.

\section{Summary and conclusions}

In this paper we have examined the flow past heated topography with both linear and nonlinear models. It was first shown that the forcing of an obstacle with horizontally homogenous surface heating can be ap- 

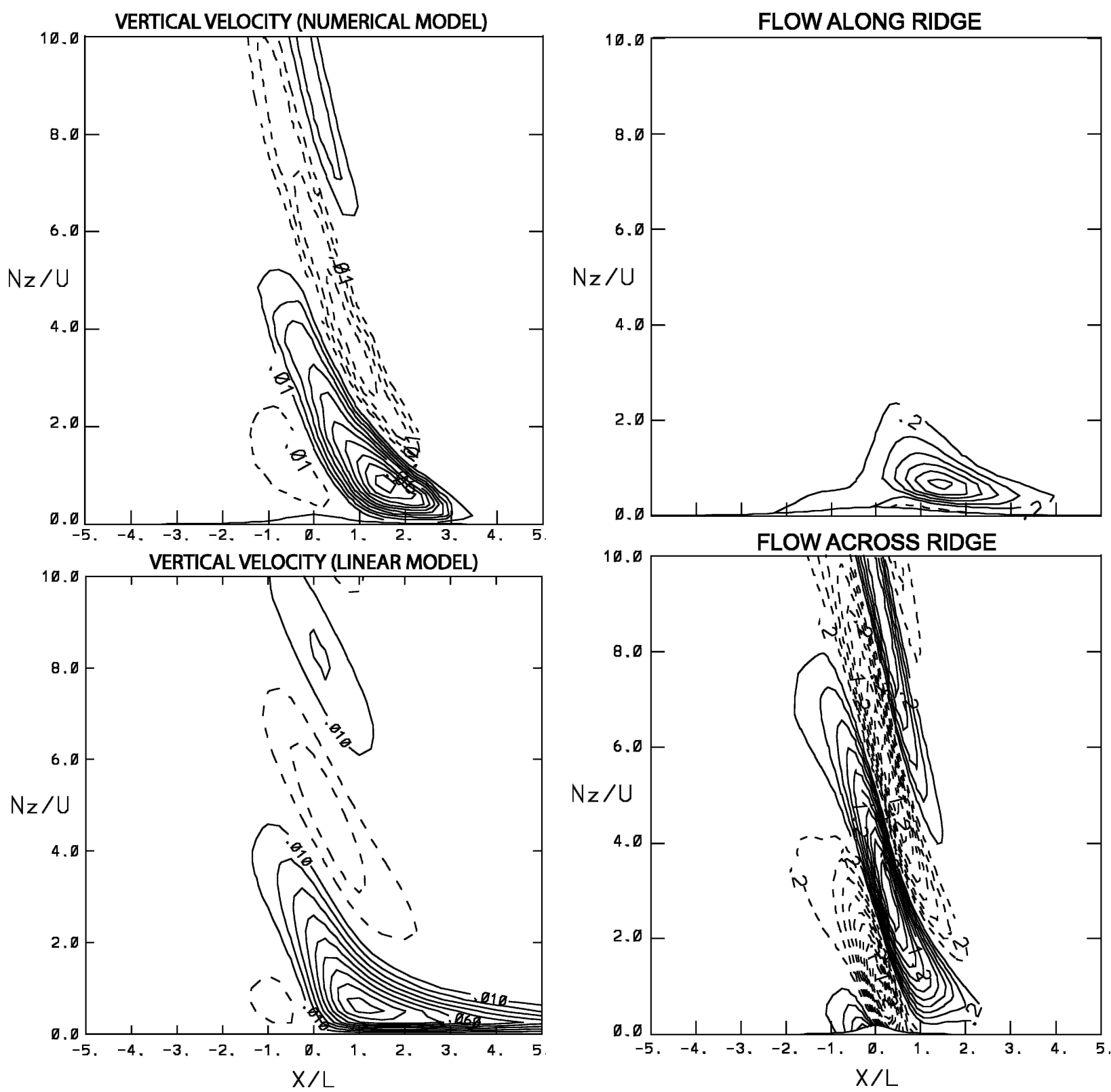

FIG. 11. Same as Fig. 10 except for $\tilde{Q}_{o}=0.1, \tilde{D}=0.5$. Thermalorographic response minus orographic response from (a) numerical simulation and (b) linear solution.

proximated by the forcing of an obstacle with surface heating isolated over the obstacle. The small-amplitude flow past an obstacle with isolated heating was then examined with a linear model. Under the linear assumption, the flow response to heated topography is simply the addition of the separate responses to thermal and orographic forcing. The individual responses were first considered separately and then combined to determine the relative importance of the two forcings. It was shown that the ratio of thermal response (rela-

FIG. 12. Full thermal-orographic response for flow (a) along and (b) across a heated elliptically shaped obstacle with $\tilde{Q}_{o}=1.0$ and $\tilde{D}=0.5$. Contour interval equals 0.2 .

tive to orographic response) could be increased by either increasing the nondimensional heating rate $\tilde{Q}_{o}$ or decreasing the nondimensional heating depth $\tilde{D}$ [where $\tilde{Q}_{o}=\left(g Q_{o} a D / \theta_{o} U^{3}\right)$ and $\left.\tilde{D}=(N D) / U\right]$. It was also shown that for the case of an elliptically shaped heated obstacle the lifting at low levels is maximized when the flow is along the major axis of the obstacle.

The predictions of the linear model were then tested in a nonlinear anelastic model. We first examined the response to orography and an isolated heat source 
separately. Very good agreement was found between the numerical simulations and the linear model for both cases. We then examined the case of uniformly heated flow past an obstacle. In these simulations, we isolated the thermal response by subtracting the orographiconly response from the full thermal-orographic response. Although the agreement with the linear model was not as good as in the previous examples, the numerical simulations were able to capture the main features of the thermal response. Finally, we examined numerical simulations of the flow along and across an elliptically shaped heated obstacle, where it was shown that the lifting is maximized when the flow is along the major axis of the obstacle.

In Part II of this study we will examine the moist convective response to heated flow over both idealized and complex topography. The complex topography chosen is that of the Rocky Mountains of the United States. This study will show that the moist convective response is maximized when the flow is aligned with major ridges in the terrain.

Acknowledgments. Reviews of an earlier draft of this manuscript by Rich Rotunno and Jimy Dudhia are gratefully acknowledged. Discussions with Joe Klemp were also very helpful.

\section{REFERENCES}

Banta, R. M., and C. Barker Schaaf, 1987: Thunderstorm genesis zones in the Colorado Rocky Mountains as determined by traceback of geosynchronous satellite images. Mon. Wea. Rev., 115, 463-476.

Byers, H. R., and R. R. Braham Jr., 1949: The Thunderstorm. U.S. Government Printing Office, 287 pp.

Clark, T. L., 1977: A small scale numerical model using a terrain following coordinate transformation. J. Comput. Phys., 24, 186-215.

Crapper, G. D., 1959: A three-dimensional solution for waves in the lee of mountains. J. Fluid Mech., 6, 51-76.

Lin, Y.-L., 1986: Calculation of airflow over an isolated heat source with application to the dynamics of V-shaped clouds. J. Atmos. Sci., 43, 2736-2751.

Queney, P., 1948: The problem of air flow over mountains: A summary of theoretical studies. Bull. Amer. Meteor. Soc., 29, $16-26$.

Raymond, D. J., 1972: Calculation of airflow over an arbitrary ridge including diabatic heating and cooling. J. Atmos. Sci., 29, 837-843.

Reisner, J. M., and P. K. Smolarkiewicz, 1994: Thermally forced low Froude number flow past three-dimensional obstacles. $J$. Atmos. Sci., 51, 117-133.

Smith, R. B., 1980: Linear theory of stratified hydrostatic flow past an isolated mountain. Tellus, 32, 348-364.

— and Y. L. Lin, 1982: The addition of heat to a stratified airstream with application to the dynamics of orographic rain. Quart. J. Roy. Meteor. Soc., 108, 353-378.

Smolarkiewicz, P. K., and R. Rotunno, 1989: Low Froude number flow past three-dimensional obstacles. Part I: Baroclinically generated lee vortices. J. Atmos. Sci., 46, 1154-1164.

Tian, W., and D. J. Parker, 2003: A modeling study and scaling analysis of orographic effects on boundary layer shallow convection. J. Atmos. Sci., 60, 1981-1991.

Tucker, D. F., and N. A. Crook, 2005: Flow over heated terrain. Part II: Generation of convective precipitation. Mon. Wea. Rev., 133, 2565-2582.

Wurtele, M., 1957: The three dimensional lee wave. Beitr. Phys. Freien Atmos., 29, 242-252. 